\title{
Rapid phenotypic change and diversification of a soil bacterium during 1000 generations of experimental evolution
}

\author{
Merry S. Riley, ${ }^{1,2}$ Vaughn S. Cooper, ${ }^{1}$ Richard E. Lenski, ${ }^{1}$ Larry J. Forney ${ }^{4}$ \\ and Terence L. Marsh ${ }^{1,3}$
}

Author for correspondence: Terence L. Marsh. Tel: +1 517432 1365. Fax: +1 5174323770. e-mail:marsht@pilot.msu.edu

1,2,3 Center for Microbial Ecology ${ }^{1}$, Department of Crop and Soil Science ${ }^{2}$ and Department of Microbiology ${ }^{3}$, Michigan State University, East Lansing, MI 48824, USA

4 Department of Biology, University of Idaho, Moscow, ID 83844, USA

\begin{abstract}
Evolutionary pathways open to even relatively simple organisms, such as bacteria, may lead to complex and unpredictable phenotypic changes, both adaptive and non-adaptive. The evolutionary pathways taken by 18 populations of Ralstonia strain TFD41 while they evolved in defined environments for $\mathbf{1 0 0 0}$ generations were examined. Twelve populations evolved in liquid media, while six others evolved on agar surfaces. Phenotypic analyses of these derived populations identified some changes that were consistent across all populations and others that differed among them. The evolved populations all exhibited morphological changes in their cell envelopes, including reductions of the capsule in each population and reduced prostheca-like surface structures in most populations. Mean cell length increased in most populations (in one case by more than fourfold), although a few populations evolved shorter cells. Carbon utilization profiles were variable among the evolved populations, but two distinct patterns were correlated with genetic markers introduced at the outset of the experiment. Fatty acid methyl ester composition was less variable across populations, but distinct patterns were correlated with the two physical environments. All 18 populations evolved greatly increased sensitivity to bile salts, and all but one had increased adhesion to sand; both patterns consistent with changes in the outer envelope. This phenotypic diversity contrasts with the fairly uniform increases in competitive fitness observed in all populations. This diversity may represent a set of equally probable adaptive solutions to the selective environment; it may also arise from the chance fixation of non-adaptive mutations that hitchhiked with a more limited set of beneficial mutations.
\end{abstract}

Keywords: Ralstonia, phenotypic radiation, diversity, mutation

\section{INTRODUCTION}

Micro-organisms have proven useful for studying the mechanisms and consequences of evolution. By virtue of their small size, fast growth rates and comparatively simple genetic systems, questions that could not be addressed with macro-fauna can be answered in a robust and statistical manner with micro-organisms (Helling et al., 1987; Dykhuizen, 1990, 1993; Lenski \& Travisano, 1994; Lenski et al., 1998; Rainey \& Travisano, 1998). Moreover, because of the resilience of some micro-

Abbreviations: FAME, fatty acid methyl ester; SEM, scanning electron microscopy; TEM, transmission electron microscopy. organisms to cryogenic preservation, a detailed historical record of an evolving population can be preserved and used comparatively in the dissection of evolutionary processes (Lenski \& Travisano, 1994).

Our present work extends previous investigations of the experimental evolution of a soil bacterium (Korona et al., 1994; Korona, 1996; Nakatsu et al., 1998). The original intent of this work was to extend investigations performed on 'domesticated' Escherichia coli to a recently isolated 'undomesticated' soil bacterium. In addition, two different environmental conditions were used as selective regimes during the propagation of lines derived from this isolate for 1000 generations. Twelve replicate populations were maintained in a liquid shake 
flask and six populations were cultivated on agar. The former provided a mass-action environment that is highly homogeneous throughout, while the latter provided a structured environment with complex gradients of nutrients as well as metabolites produced by the bacteria themselves. The soil bacterium used as the ancestral founder of all these populations is strain TFD41, which was identified as a Ralstonia species based on its 16S rRNA sequence (Nakatsu et al., 1998). Previous work described a substantial increase in competitive fitness of all 18 evolved populations, measured relative to the common ancestor in the experimental environments (Korona et al., 1994). Variations in colony morphology were noted (Korona et al., 1994), as were certain genome changes, both chromosomal and plasmid encoded (Nakatsu et al., 1998).

In this paper, we extend these observations with a systematic examination of cell morphology, substrate utilization, bile salts sensitivity, adhesion properties and fatty acid methyl ester (FAME) analysis of the evolved populations and their ancestor. Our objectives were to identify common phenotypic motifs that may indicate parallel genetic adaptations, as well as differences among populations within and between the two selective regimes. The notion of an 'adaptive landscape' (Wright, 1932, 1988) suggests that a population of organisms may have many potential evolutionary solutions to a selective challenge. It seems reasonable, too, that the diversity of potential solutions would be correlated with the complexity of the selective environment. Consistent with this postulate is the observation that variation in colony morphology was greater amongst the populations evolved on solid media than those evolved in liquid (Korona et al., 1994; Korona, 1996).

\section{METHODS}

Strains and media. Strain TFD41 was originally isolated from soil based on its ability to grow on 2,4-dichlorophenoxyacetate (Tonso et al., 1995). The isolation of streptomycin and nalidixic acid resistant mutants of TFD41 and the maintenance of 18 replicate populations for 1000 generations have been described previously (Korona et al., 1994). The ancestor, antibiotic resistant mutants and isolates from generation 1000 of the evolved populations were maintained at $-80^{\circ} \mathrm{C}$ in glycerol stocks. Cultures derived from frozen stocks were maintained on plates for no longer than 2 weeks. Cells were routinely grown on either nutrient agar or R2A agar at $30^{\circ} \mathrm{C}$ unless otherwise indicated.

Microscopy. Light microscopy was performed on a Zeiss Axioskop microscope. Cells were taken from freshly streaked nutrient agar plates that had been incubated at $30{ }^{\circ} \mathrm{C}$ for 48-72 h. The presence of outer capsule material was detected with an India ink stain (Doetsch, 1981) and representative micrographs were made at $1000 \times$ magnification with an oil immersion lens (Zeiss). Cells were evaluated for the presence of capsule as well as cell shape and mean length. Images were captured Son Kodak TMAX film.

Scanning electron microscopy (SEM) was performed on cells grown on nutrient agar plates as described for light microscopy. The cells were fixed in $4 \%$ glutaraldehyde, mounted on polylysine-coated coverslips, dehydrated in an ethanol series $(25 \%, 50 \%, 75 \%$ and $95 \%)$, critical-point dried and sputter coated with gold. At each step the cells were treated as gently as possible so as to preserve the integrity of the outer cellular architecture. The mounted cells were viewed on a JEOL scanning electron microscope at several different magnifications. Images were captured and stored electronically. At least ten fields, at approximately $2500 \times$ magnification, were viewed for each lineage to obtain a representative sample of morphology. Cell length of wellisolated cells was measured from end to end, excluding surface appendages. The mean cell length was calculated from a minimum of 25 cells in a minimum of two fields. The mean cell length of an evolved population was deemed to depart significantly from that of the ancestor if there was no overlap between the respective means \pm twice the standard error.

Transmission electron microscopy was performed on the ancestor and three evolved populations. The cells were grown as described above for light microscopy, scraped from the agar plate and pelleted by centrifugation. The pellets were resuspended in a $4 \%$ agar solution and allowed to solidify. The agar was diced into $1 \mathrm{~mm}^{3}$ squares then fixed in $2 \%$ glutaraldehyde buffered with $100 \mathrm{mM}$ sodium cacodylate ( $\mathrm{pH} 7 \cdot 2$ ) for $2 \mathrm{~h}$. The fixed cubes were washed three times in $100 \mathrm{mM}$ sodium cacodylate buffer $(\mathrm{pH} 7 \cdot 2$ ), post-fixed in $1 \%$ osmium tetroxide for $1.5 \mathrm{~h}$ and then washed four times in deionized water. The specimens were dehydrated in a graded acetone series and infiltrated in a graded Quetol resin series and embedded. Preparations were stained with uranyl acetate/lead citrate. All preparation steps were conducted at room temperature. Scanning and transmission electron microscopies were performed at the Michigan State University Center for Electron Optics.

BIOLOG assays. Carbon utilization profiles of the ancestor and evolved populations were determined using BIOLOG GN plates and the protocol provided by the vendor (modified to accommodate a Ralstonia sp.). Briefly, an aliquot of an overnight culture grown on $1 / 3 \times$ Trypticase soy broth was transferred to fresh media and cells were grown at $30^{\circ} \mathrm{C}$ in a reciprocating shaker to mid-exponential phase. Cells were harvested by centrifugation and resuspended in $0 \cdot 145 \mathrm{M} \mathrm{NaCl}$ to an of $\mathrm{OD}_{590} 1 \cdot 0$. One lineage, L16, had anomalously low cell density at an $\mathrm{OD}_{590}$ of 1.0 and therefore was adjusted to $\mathrm{OD}_{590}$ $2 \cdot 0$. Cells were then starved for $15 \mathrm{~min}$ at room temperature prior to the inoculation of the microtitre plates with $150 \mu \mathrm{l}$ cell suspension per well. Each strain was tested in duplicate. The plates were incubated at $30{ }^{\circ} \mathrm{C}$ and the $\mathrm{OD}_{590}$ measured using a Bio-Kinetics microtitre plate reader (model EL 312E, BioTek Instruments) every $2 \mathrm{~h}$ for a total of $8 \mathrm{~h}$. Plates were vigorously shaken prior to each reading and the $\mathrm{OD}_{590}$ was corrected for the zero substrate control well. Some substrates had a variable response where one of the replicates was unambiguously positive $\left(\mathrm{OD}_{590}>0 \cdot 1\right.$ after zero substrate correction) and the other borderline. These substrates are indicated (see Fig. 3).

Analysis of BIOLOG data. BIOLOG optical density (OD) readings from the plate reader were imported into a database and fed into a Microsoft Visual Basic-Excel program that approximated the area under the growth curve for each of the 95 substrates for each genotype replicate. Following the general method of Guckert et al. (1996), the program calculates a trapezoidal area that approximates the result of fitting and integrating each individual growth curve. This curve area approach collapses several values from a growth curve into a single value and integrates several properties (e.g. duration of lag, growth rate, yield). Individual values for each substrate, 

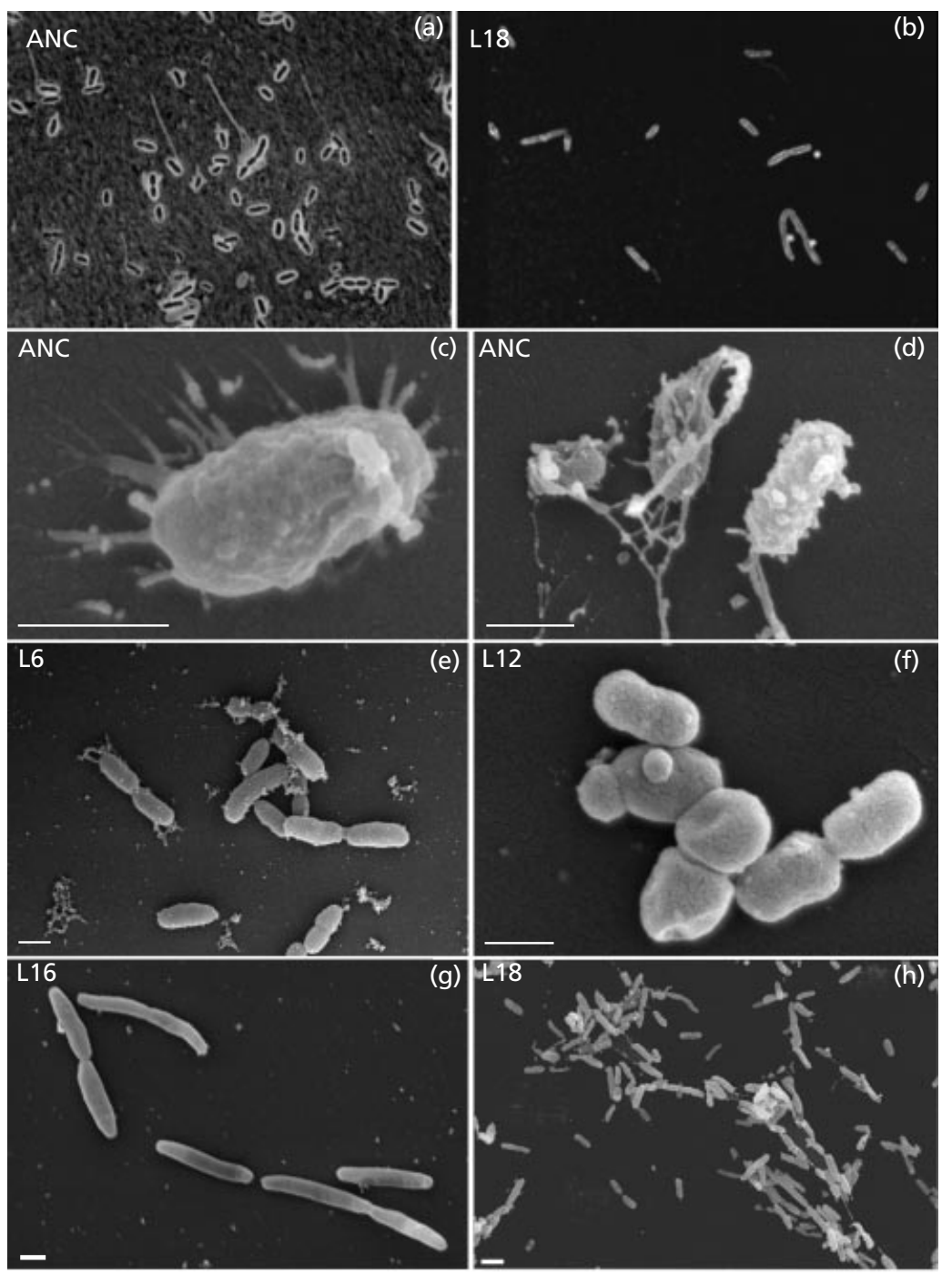

Fig. 1. Light and scanning electron microscopy of the ancestor, Ralstonia strain TFD41, and several evolved lines. (a) Light micrograph of ancestor. (b) Light micrograph of evolved L18. (c, d) Scanning electron micrographs of ancestor. (e-h) Scanning electron micrographs of evolved lines L6, L12, L16 and L18. Bars, $1 \mu \mathrm{m}$.

which we refer to as 'catabolic area', were subjected to a hierarchical cluster analysis (SYSTAT, v. 7.0, SPSS) to determine the relationships between the ancestors and the evolved lineages based on their patterns of carbon source utilization. The area data were expressed on a continuous numeric scale, and therefore normalized Euclidean distances were calculated for our clustering analysis. Ward's linkage method (Ward, 1963) was applied to adjust for covariance and to focus on mean values within clusters. Other linkage methods were also tested and none gave substantially different cluster patterns (data not shown). The source codes for the above procedures are available by request from the author (V.S.C. at cooperva@pilot.msu.edu).

Bile salts MIC. Overnight cultures grown on $1 / 3 \times$ TSB were diluted 1:100 into fresh broth containing a range of bile salts (Difco) concentrations $\left(0 \cdot 1 \mathrm{~g} \mathrm{l}^{-1}\right.$ to $\left.20 \mathrm{~g} \mathrm{l}^{-1}\right)$. The cultures were incubated at $30^{\circ} \mathrm{C}$ and scored for growth at 24,48 and $72 \mathrm{~h}$. Any increase in turbidity above the initial OD was scored as a positive. All evolved populations were tested in duplicate, and no differences were detected between these replicates.

Adhesion. The adhesion assay was based on the retention of bacterial cells in a saturated sand matrix supported in a column and has been described previously (DeFlaun et al.,
1990). Briefly, $A+N$ minimal medium (Wyndham, 1986) supplemented with $30 \mathrm{mM} \mathrm{L}$-aspartic acid (AN-asp) as the sole carbon source was inoculated with the ancestor or an evolved population from a fresh nutrient agar plate and grown overnight at $30^{\circ} \mathrm{C}$ in a reciprocating shaker. Prior screening indicated that the ancestor and all 18 evolved lineages could utilize L-aspartic acid. The culture was diluted into fresh ANasp and grown to late-exponential phase. The cells were harvested by centrifugation, resuspended in phosphate buffered saline (PBS) and adjusted by dilution with PBS to an $\mathrm{OD}_{590}$ of $0 \cdot 5-0 \cdot 6$ (1 cm light path).

Bio-Rad Econocolumns $(5 \times 1.5 \mathrm{~cm}$ inner diameter $)$ were packed with $11 \mathrm{~g}-50+70$ mesh sand (Sigma S-9887) and rinsed with approximately $15 \mathrm{ml}$ PBS. The cell suspension $(3.5 \mathrm{ml})$ was pipetted gently onto the column, an entire pore volume was allowed to pass into the matrix $(3.5 \mathrm{ml}$ void volume) and then flow was stopped. The loaded column was incubated undisturbed at room temperature for $1 \mathrm{~h}$ to allow cells to bind to the matrix. The column was then eluted with $14 \mathrm{ml}$ PBS, collected in four fractions. The fraction of cells eluted was determined spectrophotometrically in a $1 \mathrm{~cm}$ path length quartz cuvette (Hewlett Packard 8452A Diode Array Spectrophotometer) and the fraction of cells bound to the column was calculated as fraction bund $=1-$ total OD 
Table 1. Morphological characteristics of the evolved populations

Minimal medium with 2,4-dichlorophenoxyacetate as the carbon source was used for selection (see Methods).

\begin{tabular}{|c|c|c|c|c|c|c|}
\hline \multirow[t]{2}{*}{ Strain" } & \multirow[t]{2}{*}{ Selection } & \multirow{2}{*}{$\begin{array}{c}\text { Colony } \\
\text { type } †\end{array}$} & \multirow{2}{*}{$\begin{array}{c}\text { Light } \\
\text { microscopy } \neq\end{array}$} & \multicolumn{3}{|c|}{ SEM } \\
\hline & & & & Cell surface & $\begin{array}{l}\text { Mean cell length } \\
(\mathrm{nm}) \mathbb{S}\end{array}$ & $2(\mathrm{SE}) \|$ \\
\hline Ancestor & Soil & + & $\mathrm{C}+\mathrm{P}++$ & ++ Capsule & 1.95 & $0 \cdot 14$ \\
\hline L1, Str & Liquid & - & $\mathrm{C}-\mathrm{P}-$ & Smooth & $1 \cdot 8$ & $0 \cdot 12$ \\
\hline L2, Nal & Liquid & - & $\mathrm{C}-\mathrm{P}-$ & Smooth & $2 \cdot 959$ & $0 \cdot 59$ \\
\hline L3, Str & Liquid & \pm & $\mathrm{C}-\mathrm{P}-$ & Smooth & $5 \cdot 649$ & $0 \cdot 92$ \\
\hline $\mathrm{L} 4, \mathrm{Nal}$ & Liquid & + & $\mathrm{C}-\mathrm{P}+$ & \pm Capsule & $1 \cdot 399$ & $0 \cdot 09$ \\
\hline L5, Str & Liquid & + & $\mathrm{C}-\mathrm{P}+$ & Smooth & $4 \cdot 089$ & $0 \cdot 68$ \\
\hline L6, Nal & Liquid & - & $\mathrm{C}-\mathrm{P}-$ & Smooth & 1.469 & $0 \cdot 13$ \\
\hline L7, Str & Liquid & - & $\mathrm{C}-\mathrm{P}-$ & Smooth & $3 \cdot 449$ & $0 \cdot 52$ \\
\hline L8, Nal & Liquid & - & $\mathrm{C}-\mathrm{P}-$ & \pm Capsule & $3 \cdot 219$ & $0 \cdot 46$ \\
\hline L9, Str & Liquid & - & $\mathrm{C}-\mathrm{P}-$ & Smooth & $5 \cdot 199$ & $0 \cdot 81$ \\
\hline L10, Nal & Liquid & - & $\mathrm{C}-\mathrm{P}-$ & \pm Capsule & 3.599 & $0 \cdot 75$ \\
\hline L11, Str & Liquid & - & $\mathrm{C}-\mathrm{P}-$ & Smooth & $3 \cdot 239$ & $0 \cdot 47$ \\
\hline L12, Nal & Liquid & - & $\mathrm{C}-\mathrm{P}-$ & Smooth & $1 \cdot 88$ & $0 \cdot 16$ \\
\hline L13, Str & Agar & + & $\mathrm{C}-\mathrm{P}+$ & \pm Capsule & $4 \cdot 539$ & $0 \cdot 64$ \\
\hline L14, Nal & Agar & - & $\mathrm{C}-\mathrm{P}-$ & \pm Capsule, blebs & $1 \cdot 97$ & $0 \cdot 34$ \\
\hline L15, Str & Agar & \pm & $\mathrm{C}-\mathrm{P}-$ & \pm Capsule & 1.519 & $0 \cdot 08$ \\
\hline L16, Nal & Agar & - & $\mathrm{C}-\mathrm{P}-$ & Smooth & $9 \cdot 149$ & $2 \cdot 73$ \\
\hline L17, Str & Agar & - & $\mathrm{C}-\mathrm{P}-$ & Smooth & $4 \cdot 089$ & $0 \cdot 7$ \\
\hline L18, Nal & Agar & \pm & $\mathrm{C}-\mathrm{P}+$ & Smooth, blebs & $1 \cdot 93$ & $2 \cdot 07$ \\
\hline
\end{tabular}

*Str, streptomycin resistant; Nal, nalidixic acid resistant.

$\dagger+$, Highly mucoid; \pm , intermediate mucoid; - , non-mucoidal.

$\ddagger \mathrm{C}+$, detectable capsule with India ink; $\mathrm{C}-$, capsule not detectable with India ink; $\mathrm{P}++$, prostheca-like appendages present in greater than $75 \%$ of cells; P , prostheca-like appendages present in less than $25 \%$ of cells; P-, prostheca-like appendages absent.

$\$$ Mean of at least 25 cells from a minimum of two SEM fields; a total of ten fields of each population were viewed.

$\|$ Standard error $(\mathrm{SE})=$ standard deviation $/(\sqrt{ } n)$.

S Statistically different from the ancestor.

eluted/total OD loaded. All populations were tested in triplicate.

FAME analysis. This was conducted on a subset of the derived lineages (L1, L5, L6, L8, L9, L11, L12, L16, L17 and L18), the original ancestor as well as the $\mathrm{Str}^{\mathrm{R}}$ and $\mathrm{Nal}^{\mathrm{R}}$ derivatives of the ancestor. Strains were streaked from $-80^{\circ} \mathrm{C}$ stocks onto $1 / 3 \times$ Trypticase soy agar, incubated at $30^{\circ} \mathrm{C}$ and harvested after $2 \mathrm{~d}$ by scraping the plates with a sterile spatula directly into dry-heat-sterilized glass culture tubes. The resulting cell pellet was stored at $-80^{\circ} \mathrm{C}$ until assayed. Each sample was extracted and run in triplicate as described previously by Sasser (1997). FAME analysis was performed on a Hewlett Packard 5890 series II gas chromatograph equipped with a FID detector. A Hewlett Packard Ultra2 column (cross-linked $5 \%$ phenyl methyl silicone) of $25 \mathrm{~cm} \times 0.22 \mathrm{~mm}$ with $0.33 \mu \mathrm{m}$ film thickness was used with ultra-high purity hydrogen as the carrier gas at $50 \mathrm{ml} \mathrm{min}{ }^{-1}$ using a 50:1 split ratio. The initial temperature, $170{ }^{\circ} \mathrm{C}$, was ramped to $270{ }^{\circ} \mathrm{C}$ at a rate of $5{ }^{\circ} \mathrm{C}$ $\min ^{-1}$. The column was baked for $2 \mathrm{~min}$ at $300^{\circ} \mathrm{C}$ after each run. The injector and detector temperatures were maintained at $250{ }^{\circ} \mathrm{C}$ and $300^{\circ} \mathrm{C}$, respectively. FAME data were analysed with the Sherlock MIDI system and the Dendrogram program (MIDI). The former identifies the closest microbial relative based on the fatty acid profiles, while the latter compares the lineages based on a clustering analysis using unweighted pairgroup method with arithmetic averages (UPGMA).

\section{RESULTS}

\section{Microscopy}

One of the first phenotypic changes noted in the evolved populations was colony morphology (Korona et al., 1994). Because these differences were suggestive of changes in capsule production, the cell morphology of the ancestor and evolved populations was systematically assessed using both light and electron microscopy. Fig. 1 (a) shows the cell morphology of the ancestor visualized with a capsule counter-stain (India ink). Cells of the ancestral strains were heavily encapsulated, as evidenced by the light-bright halo surrounding the cells. In addition, very long prostheca-like appendages were present on most cells. All of the evolved populations showed a substantial reduction in encapsulation as judged by light microscopy. Four lineages (L4, L5, L13 and L18) showed some indication of prostheca-like appendages but the length of the appendages and the 


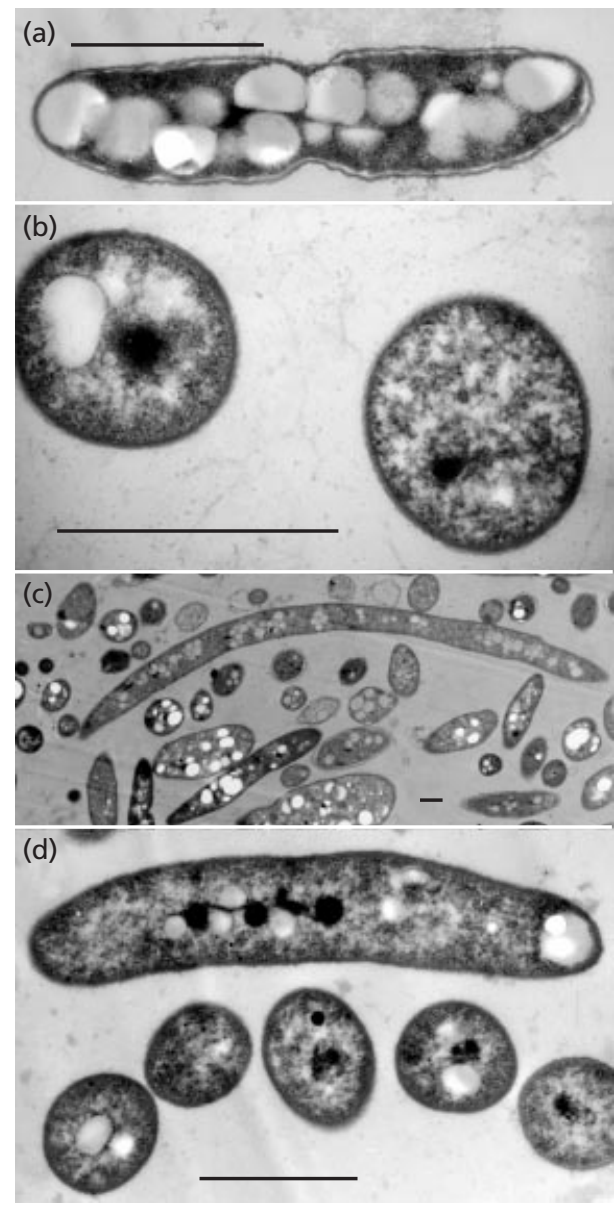

Fig. 2. Transmission electron micrographs of the ancestor and two evolved lines, L16 and L18. (a, b) Ancestor. (c) Evolved line L16. (d) Evolved line L18. Bars, $1 \mu \mathrm{m}$.

number of cells with appendages were considerably less than for the ancestor. Several lineages had cells that were consistently longer than the ancestor. Cells from population L18 are shown in Fig. 1(b). L18 is an example not only of the lack of encapsulation but also of the occasional presence of vestigial prosthecae. Light-bright 'blebs' were occasionally seen associated with the cell surface or with a prostheca-like appendage, as can be seen in this photomicrograph. The compiled data are presented in Table 1.

Scanning electron micrographs of the ancestor and four evolved populations are also shown in Fig. 1. The ancestor (Fig. 1c and d) presented a complex contoured surface with clear indications of heavy encapsulation as well as prostheca-like appendages. The appendages were not as long as those observed using light microscopy, perhaps due to the more rigorous preparative steps required by SEM. SEM micrographs of evolved populations L6, L12, L16 and L18 are shown in Fig. 1(e-h). These lineages do not display evidence of encapsulation. Cells from the evolved populations L12 and L18 do not vary significantly in length from the ancestor (Table 1 ).
L16 was remarkable in that its mean cell length was 4.7 times the mean length of the ancestor and occasional cells of 20-25 $\mu \mathrm{m}$ length were observed. Frequently, one terminus of the rod-shaped cell had greater light and electron opacity than the other. L18 showed evidence of vestigial prostheca-like appendages, some evidence of capsule material, and the unusual light-bright blebs mentioned above. L6 is one of three lineages that was significantly shorter than the ancestor. Eight of the 12 lineages evolved in liquid and three of six evolved on agar had significant increases in mean cell length, ranging from 1.5 to 4.7 times that of the ancestor. No significant differences between the original ancestor and the streptomycin or nalidixic acid resistant mutants were detected with microscopy. Table 1 gives the mean cell lengths for all of the lineages based on SEM.

Transmission electron microscopy (TEM) was performed to confirm the differences in morphology between the ancestor and two lineages, and these images are shown in Fig. 2. As viewed with TEM, L16 and L18 (Fig. 2c and d, respectively) had internal cellular details similar to those seen in the ancestor (Fig. 2a and b), including structures consistent with poly- $\beta$-hydroxybutyrate storage granules. The TEM images also confirm the integrity of the cytoplasm throughout the length of the unusually long cells of L16.

\section{BIOLOG}

To monitor changes in the catabolic breadth of the replicate lines, BIOLOG GN plates testing the ability to metabolize 95 different carbon sources were used. The salient features of the BIOLOG data (Fig. 3) are as follows. First, the ancestor was capable of utilizing 43-49 of the 95 substrates in the BIOLOG GN plates in a pattern consistent with the type strain description of Ralstonia eutropha (formerly Alcaligenes eutrophus; Krieg \& Holt, 1984). Second, no two derived genotypes had the same pattern of carbon utilization. Third, the greatest range of utilization patterns appeared amongst the populations evolved on agar. Fourth, while there was tremendous phenotypic variation among the evolved populations, there was one discernible pattern detected among the lineages evolved in liquid as described below.

The results of hierarchical cluster analysis on the BIOLOG data using all 18 lineages evolved either in liquid or on solid media are presented in Fig. 4. Note that the antibiotic resistance marker determines the primary grouping for the lineages evolved on liquid. Lineages founded with the streptomycin-resistant ancestor (L1, L3, L5, L7, L9 and L11) group with the original ancestor and the two antibiotic resistant variants whereas those founded with the nalidixic acid resistant strain (L2, L4, L6, L8, L10 and L12) group separately. No such ancestor-dependent clustering is seen among the lineages evolved on agar. This marker effect was examined statistically by two $t$-tests: one which divided all evolved populations based on their antibiotic marker state, and another which only divided 


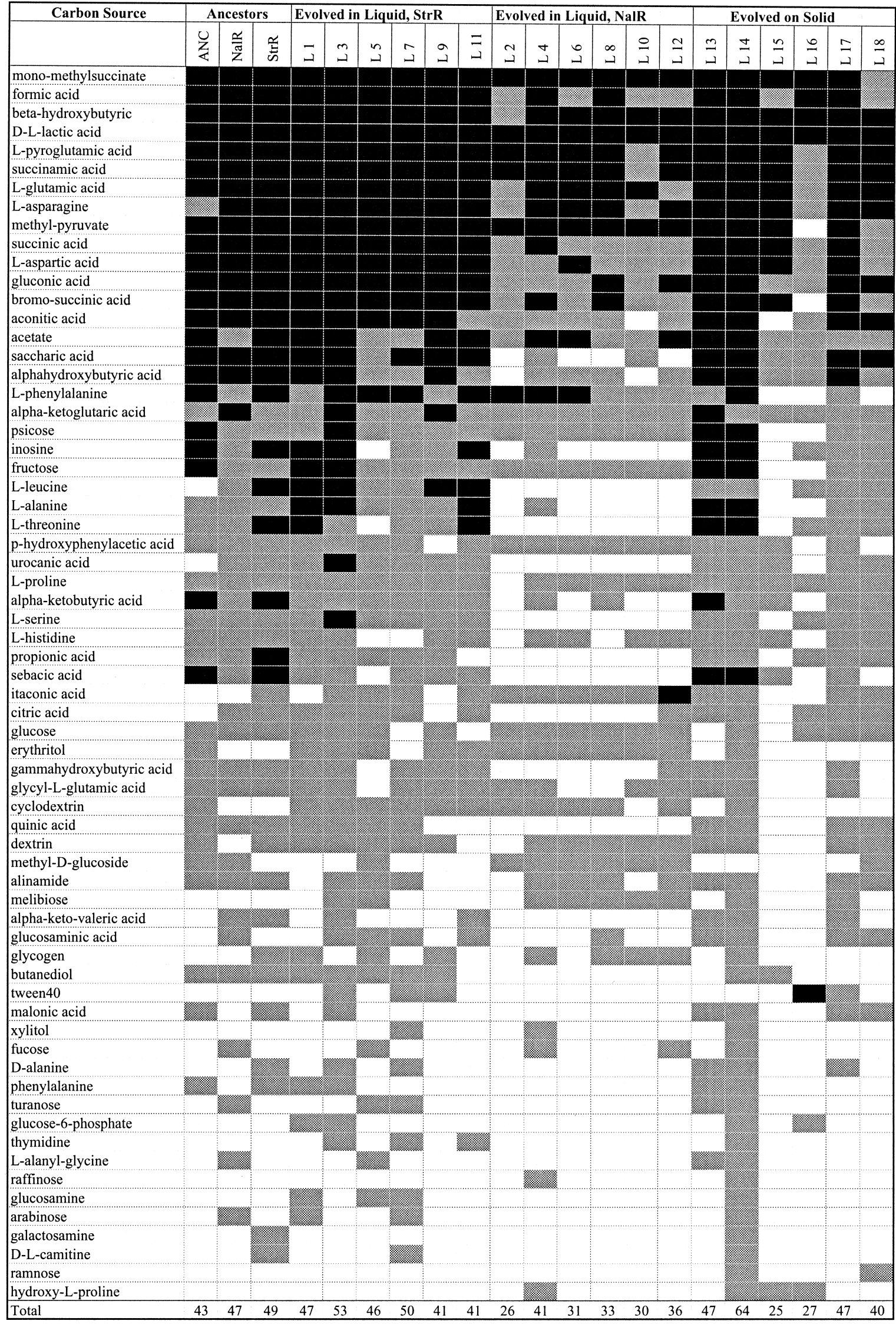

Fig. 3. For legend see facing page. 


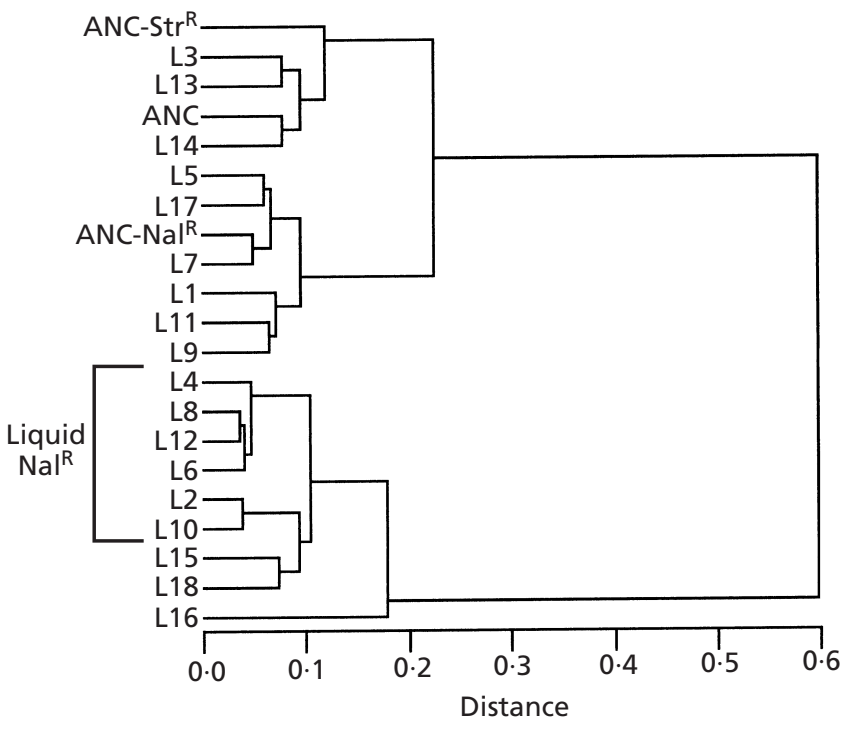

Fig. 4. Clustering based on catabolic profiles of the 18 evolved lines, their two proximate ancestors that differ in antibiotic resistance markers (ANC-Str ${ }^{R}$ and ANC-Nal ${ }^{R}$ ), and the original Ralstonia strain TFD41 (ANC). The tree was constructed by hierarchical cluster analysis based on the method of Ward (1963), adjusted for covariance, using SYSTAT v. 7.01. See Table 1 for strain identification. ANC indicates the original Ralstonia strain TFD41, while ANC-Str ${ }^{R}$ and ANC-Nal ${ }^{R}$ denote antibiotic resistance marker variants thereof.

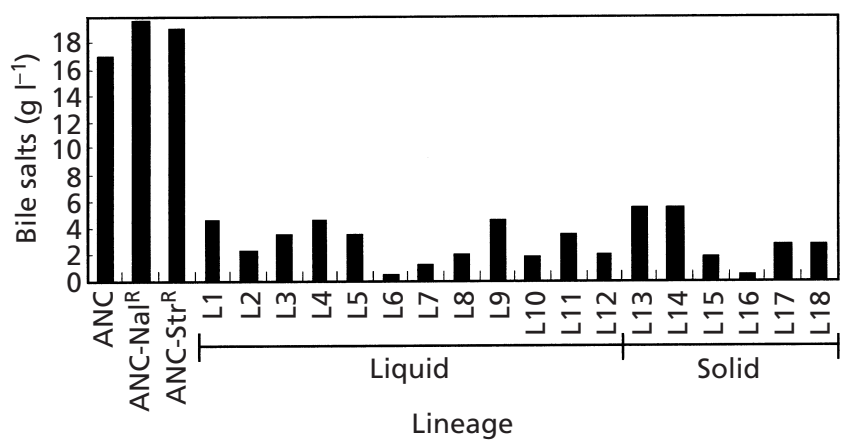

Fig. 5. MIC of bile salts. Each strain was tested in duplicate. The MIC was defined as the concentration at which no detectable increase in turbidity was observed after $5 \mathrm{~d}$ at $30^{\circ} \mathrm{C}$. ANC indicates the original Ralstonia strain TFD41, while ANC-Str ${ }^{R}$ and ANC-Nal ${ }^{R}$ denote antibiotic resistance marker variants thereof.

liquid-evolved populations. We found that $\mathrm{Nal}^{\mathrm{R}}$ populations had diminished breadth in carbon source utilization compared to the $\operatorname{Str}^{\mathrm{R}}$ populations, but the

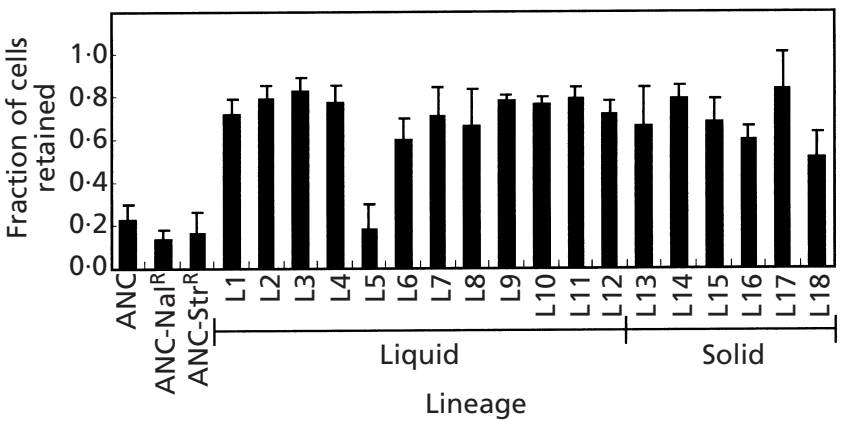

Fig. 6. Adhesion of ancestor and derived genotypes to a sand substrate. Cells grown on L-aspartic acid were incubated in the sand matrix for $1 \mathrm{~h}$. Following elution, the fraction of cells not attached to the sand was determined spectrophotometrically and the fraction retained was calculated by subtraction. All assays were performed in triplicate. Error bars indicate standard deviation. ANC indicates the original Ralstonia strain TFD41, while ANC-Str ${ }^{R}$ and ANC-Nal ${ }^{R}$ denote antibiotic resistance marker variants thereof.

difference was only clearly significant among the liquidevolved populations (all evolved lines, $t=1 \cdot 91$, d.f. $=$ $16, P=0.074$; liquid evolved only, $t=3.59$, d.f. $=10$, $P=0.005$; both two-tailed tests).

\section{MIC of bile salts}

In Gram-negative bacteria, changes to the outer envelope can alter sensitivity to antibiotics and detergents. To determine if any of the evolved populations changed in this respect, the MIC of bile salts was determined (Fig. $5)$. The ancestor and its antibiotic-resistant mutants were able to grow in media with bile salts concentrations above $16 \mathrm{~g} \mathrm{l}^{-1}$. In contrast, all 18 evolved populations were substantially more sensitive, with growth inhibition observed at concentrations below $6 \mathrm{~g} \mathrm{l}^{-1}$ in all cases.

\section{Adhesion}

The ancestors and evolved populations displayed very different adhesion properties (Fig. 6). All of the evolved strains, with one exception (L5), had high affinity for the sand matrix, which retained more than $50 \%$ of the cells applied to the column. The ancestral strains, on the other hand, did not adhere strongly to the sand, with about $80 \%$ of the cells loaded onto the column passing through the sand matrix. L5, the only exception among the evolved populations, behaved like the ancestor in that $80 \%$ of cells applied to the column were recovered in the effluent.

Fig. 3. Carbon utilization patterns of the ancestor, $\mathrm{Str}^{\mathrm{R}}$ and $\mathrm{Nal} \mathrm{I}^{\mathrm{R}}$ mutants thereof, and the 18 evolved lines after 1000 generations. Black cells indicate the carbon source was consistently metabolized; grey cells indicate variability in the catabolism of the particular carbon source across replicate BIOLOG plates. Carbon sources were sorted first by overall mean (mean performance of all lineages on a particular substrate), in descending order, and then by coefficient of variation (the standard deviation divided by the overall mean), in descending order. The total number of carbon sources used by each strain is shown on the bottom row, including both consistently positive and variable carbon sources. The lines that evolved in liquid media are grouped by their ancestral antibiotic resistance markers. 


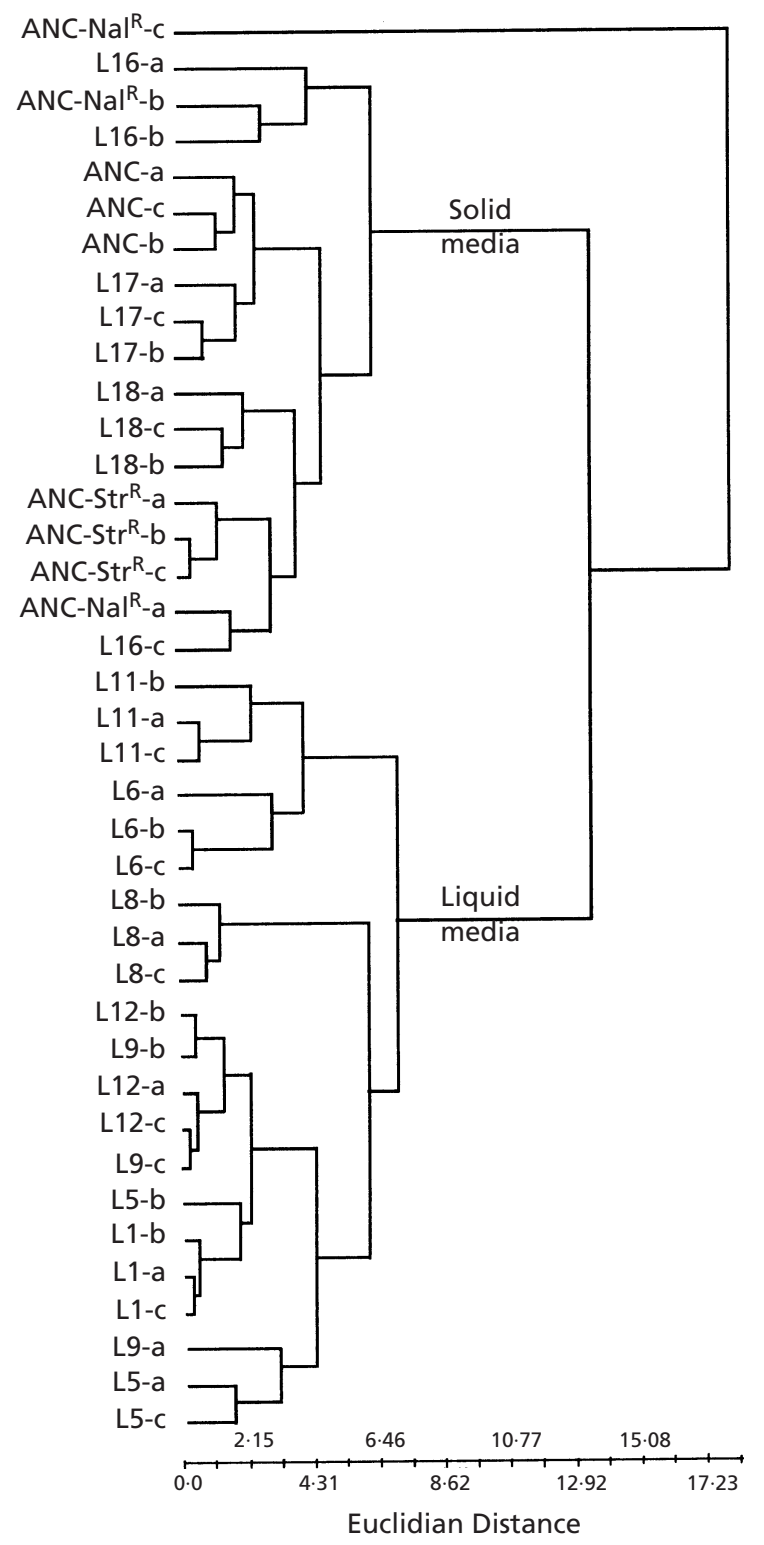

Fig. 7. Cluster analysis of FAME data from the ancestral strains and ten evolved lineages. L1, L5, L6, L8, L9, L11 and L12 evolved in liquid medium, whereas L16, L17 and L18 evolved on agar. Each strain was tested in triplicate (denoted $a, b$ and c). ANC indicates the original Ralstonia strain TFD41, while ANC-Str ${ }^{R}$ and ANC-Nal ${ }^{R}$ denote antibiotic resistance marker variants thereof.

\section{FAME analysis}

FAME analysis of the ancestors and a subset of ten evolved lineages consistently revealed nine peaks corresponding to fatty acids $14: 0,14: 03-\mathrm{OH} / 16: 1$ iso I, $16: 1 \omega 7 c / 15$ iso $2-\mathrm{OH}, 16: 0,17: 0$ cyclo, $16: 02-\mathrm{OH}$, $18: 1 \omega 9 c / \omega 12 t / \omega 7 c, 18: 0$ and $18: 12-\mathrm{OH}$. Note that in this nomenclature a slash indicates that two or more fatty acids co-elute under these conditions and they are indistinguishable from one another. MIDI analysis indicated that all lineages resemble Pseudomonas pickettii or Burkholderia cepacia most closely among those organisms in the database. A cluster analysis of these data placed the ancestral strains and the lineages evolved on solid medium together in one group that was distinct from the lineages evolved in liquid (Fig. 7). The reproducibility of this major division is quite high, as indicated by the frequent clustering of the three replicates for each population. Only the $\mathrm{Nal}^{\mathrm{R}}$ parental type showed variability in this regard. The distinguishing features in the FAME profiles that account for the clustering were the relative areas of three peaks that correspond to between three and six fatty acids. The $18: 1 \omega 7 c / \omega 9 t / \omega 2 t$ peak was dominant in the liquidmedium derived lineages, whereas $16: 1 \omega 7 c / 15$ iso 2$\mathrm{OH}$ and 16:0 were the dominant peaks in the ancestor and in those lineages evolved on agar.

\section{DISCUSSION}

After 1000 generations of evolution of Ralstonia strain TFD41 in two different selective environments, we observed several different patterns of phenotypic evolution, depending on the particular trait: (1) changes common to most or all derived lines, indicating parallelism; (2) changes that were specific to the particular selective environment; (3) changes that depended upon which particular antibiotic-resistant variant of the ancestor was used as the founder, indicating historical contingency; and (4) changes that appeared more stochastic and led to phenotypic diversity among the evolved populations.

Morphological changes in the outer cell envelope occurred in all 18 evolved lineages, including reductions in the capsule (Table 1), increased sensitivity to bile salts (Fig. 5), and with one exception, increased cell adhesion to a sand matrix (Fig. 6). A genomic change also occurred in all of the evolved lineages, including a deletion of a genomic region detected by the loss of a REP-PCR fragment (Nakatsu et al., 1998); it will be interesting to determine whether these parallel genetic and phenotypic changes are related to one another. Changes that were specific to the particular selective environment were evident in the FAME profiles (Fig. 7), as well as the observation that competitive fitness was more variable among the agar-evolved lineages than among the liquid-evolved populations (Korona et al., 1994). Evidence for historical contingency was found in the carbon utilization patterns, which grouped the liquid-evolved populations as a function of the antibiotic-resistance marker borne by their immediate ancestors (Fig. 4). Finally, substantial phenotypic diversity of cell morphology and carbon utilization patterns was observed across all of the evolved populations, irrespective of differences in their selective environments or parental genotype. For example, L16 and L18 were both evolved on agar, and both were founded by the $\mathrm{Nal}^{\mathrm{R}}$ parental strain. L18 remained similar in mean cell length (Table 1) and carbon utilization profile (Fig. 3) to the ancestor, whereas L16 has increased almost five-fold in mean length and has a substantially narrowed catabolic profile. 
In broad outline, but not in detail, this mixture of parallel and divergent patterns is similar to that seen in a long-term evolution experiment with 12 replicate populations of E. coli, each propagated in a defined glucose medium for thousands of generations (Lenski, 1995; Lenski et al., 1998). In that study, all the lines improved in fitness to a similar degree when in competition against their ancestor in the glucose medium (Lenski \& Travisano, 1994). However when the competitiveness of derived lines was tested against the ancestor on different substrates, their performance was much more variable, which implies a diversity of underlying physiological changes (Travisano \& Lenski, 1996). All 12 lines also increased their mean cell volume, but the degree to which volume increased and the resulting cell shape were variable (Lenski \& Mongold, 2000). Substantial differences among the lines in genomic mutation rates also arose, as several lines evolved defects in methyl-directed mismatch repair (Sniegowski et al., 1997) while other lines exhibited bursts of activity of certain IS elements (Papadopoulos et al., 1999). However, unlike the present study with Ralstonia, in which resistance mutations used as genetic markers in the ancestor influenced the path of subsequent evolution, none of the differences among E. coli lines was strongly affected by the arabinose-utilization marker that was used to discriminate between those lines. Also, the main long-term E. coli experiment used only a single selective environment; however, when new lines derived from these original populations evolved in different thermal regimes, there emerged systematic differences among them (Lenski, 1995; Mongold et al., 1996).

In considering the many changes to Ralstonia that have accrued over 1000 generations, it is worthwhile to consider in some detail the observed alterations to the outer envelope. The outer envelope is the first part of the cell to interact with the external environment, serving both as a barrier to unwanted factors (viruses, antibiotics, etc.) and as a conduit for essential resources. Components of the outer envelope may therefore be the most sensitive and responsive cellular constituents to the selective environment. As Parke et al. (2000) put it: 'The cell surface is where the rubber hits the road in bacterial evolution.' The components of the outer envelope influence shape, chemical resistance, adhesion and metabolic properties of cells, and indeed all these properties were substantially altered during 1000 generations of evolution in Ralstonia strain TFD41. However, the underlying genetic and physiological bases of these changes are not yet sufficiently understood to interpret unambiguously the selective advantages conferred by these traits, either in the laboratory experiment or in nature. In the following paragraphs, we discuss some possible adaptive scenarios.

\section{Morphological changes}

Both light and electron microscopy clearly show that all the evolved populations have changed from the ancestral morphology in terms of the cell-surface architecture and, in many cases, cell length (Table 1). Most populations have lost all prostheca-like appendages and all experienced either partial or complete loss of their capsule. Variation in capsule production in bacteria is not uncommon (Roberts, 1996; see also Fletcher, 1996; Whitfield \& Valvano, 1993) and has been cited as an induced response that may confer an advantage in appropriate environments. In E. coli, for example, the expression of capsule material is increased when cells are subjected to an arid environment, thereby providing protection against desiccation (Ophir \& Glutnick, 1994). The cell surface also determines adhesive properties of the cell. A capsule can promote or prevent adhesion depending upon the relative chemistries of the capsule and substratum. Various extracellular polymers, including polysaccharides, that promote adhesion have been identified (Roberts, 1996). One study identified two types of extracellular polymers in the same organism: one that promotes attachment and the other that promotes detachment (Wrangstadh et al., 1990). Nonadhesive variants of Pseudomonas fluorescens that possess a capsule have also been described (Williams \& Fletcher, 1996). Simoni et al. (1998) speculate that heterogeneity within bacterial populations expressed at the level of the outer envelope can influence their transport in groundwater aquifers. Among the evolved populations of Ralstonia in this study all but one showed a substantial increase in adhesion to a sand matrix in comparison to the ancestor. Inasmuch as all of the evolved populations show reduced encapsulation, the capsule of the ancestral soil-dwelling Ralstonia strain must have served some function other than providing a matrix for adhesion to silicates. Another possible benefit for capsule-bearing strains is resistance to anti-bacterial compounds (Nikaido, 1996). Consistent with this possibility is the observation that, in a selective environment lacking such compounds, all 18 populations evolved increased sensitivity to bile salts (Fig. 5). Hence, in Ralstonia, the primary benefit of the capsule in the soil environment may be protection against chemicals and desiccation rather than adhesion to soil particles.

Perhaps the most striking phenotypic changes were in the mean cell length: 14 of 18 evolved lines showed significant changes in cell length. Of the 12 liquidderived populations, eight are significantly longer than the ancestor and two are shorter. The six lines evolved on agar also include both shorter (one of six) and longer (three of six) morphologies, including lineage L16 that increased its mean length almost fivefold. The unusually long cells of this population were not caused by incomplete septation, as TEM clearly showed a normal cytoplasm (Fig. 2). Changes in cell length have been correlated with survival strategies including, for example, the avoidance of predation in the case of long filaments (Hahn et al., 1999; Jürgens et al., 1999), but it is unclear what factors promoted such conspicuous changes in this study. The fact that some lineages evolved greater cell length and others became smaller, while all showed substantial improvements in competitive fitness (Korona et al., 1994), suggests that cell 
length was not itself a direct target of selection in these experiments.

\section{Biochemical changes}

Mechanisms for recognition and transport of nutrients also have essential components in the cell envelope. Mutations that alter either specific transport complexes or their supporting structural matrix could change carbon utilization patterns. For example, in E. coli at least 90 genes are required for biosynthesis of the outer membrane and capsule which in turn provides structural scaffolding for the products of at least 37 genes encoding integral outer-membrane proteins (see for example the E. coli genome database at http://mbgd.genome.ad.jp). If the absence of capsule is a selected phenotype, as suggested by the loss of capsule in all lineages, then any mutation in the biosynthetic pathways of outer envelope synthesis might contribute the loss of capsule and the resulting increase in fitness. Given the structural and biochemical complexity of the outer envelope, a number of possible genotypic pathways leading to loss of the capsule exist. The complex and variable carbon utilization profiles of the evolved populations are consistent with this scenario. Several populations have much narrower profiles than their ancestors, including all the lines founded from the $\mathrm{Nal}^{\mathrm{R}}$ ancestor and evolved in liquid as well as two lines evolved on agar surfaces (L15 and L16), whereas another surface-evolved line (L14) has a much broader profile than its ancestor (Fig. 3).

The capsule has been viewed as a molecular sieve that can influence the accessibility of a substrate to the cell (Nikaido, 1996). Given the diverse and complex changes in morphology and surface architecture (Fig. 1 and Table 1), it is easy to imagine that carbon utilization patterns would be somehow affected, but difficult to discern any simple association between these classes of data. We did detect the acquisition of catabolic activity (Fig. 3). In particular the ability to metabolize Tween 40 was detected in several lineages. It seems likely that the loss of the copious capsule produced by the ancestor permitted increased access of some carbon sources to existing metabolic capabilities of the cell (Nikaido, 1996). Indeed, the loss of capsule may facilitate the assimilation of the substrate 2,4-dichlorophenoxyacetate on which the replicate lines were evolved.

There is, however, one striking pattern in the carbon utilization profiles. In particular, a significant clustering was detected amongst the 12 liquid-evolved populations (Fig. 4), in which the two dominant clusters derive from the two different antibiotic-resistant ancestors that were each used to found half of the evolving lines (Korona et al., 1994). In particular, all of the liquid-evolved populations that were founded with the $\mathrm{Nal}^{\mathrm{R}}$ ancestor had much narrower catabolic profiles than did those founded by the $\operatorname{Str}^{\mathrm{R}}$ ancestor (Fig. 3). It is important to emphasize that this narrower profile was not directly attributable to the mutation that conferred $\mathrm{Nal}^{\mathrm{R}}$, because the $\mathrm{Nal}^{\mathrm{R}}$ ancestor had a catabolic breadth comparable to the common ancestor. Moreover, this association with the founding ancestor was not seen with the agar-evolved lines. Interestingly, all six of the lines that were initially $\mathrm{Nal}^{\mathrm{R}}$ and evolved in liquid reverted to sensitivity to nalidixic acid during the evolution experiment, whereas all three surface-evolved lines retained the $\mathrm{Nal}^{\mathrm{R}}$ phenotype (Korona et al., 1994). These data collectively indicate that the initial resistance marker influenced subsequent evolution in the liquid environment, although the reasons for this remain speculative. In E. coli, $\mathrm{Nal}^{\mathrm{R}}$ phenotypes can be produced by mutations in the gyrB locus that encodes DNA gyrase (Yamagishi et al., 1986) as well as by mutations in other loci that cause reduced permeability of the outer membrane (Hrebenda et al., 1985). If the nalidixic acid resistance of the ancestral strain was expressed at the level of the cell envelope, then a subsequent evolutionary change in this structure (of the sort observed in the evolved lines) could negate the phenotypic expression of the resistance mutation.

FAME analysis indicated that the liquid-evolved lineages changed from the ancestral composition, whereas the surface-evolved lines have not (Fig. 7). In particular, all six of the liquid-evolved lines that were tested had changes in the relative amounts of three elution peaks, corresponding to three to six co-eluting fatty acids, compared to the ancestors and the three tested lineages that had evolved on solid media. While these patterns are consistent, it is not obvious how these alterations relate functionally to the two different selective regimes, except perhaps to note that the greater deviation of the liquid-evolved populations from the ancestral state suggests that this regime was more different from the natural environment than was the surface regime. It is also interesting to point out that a Euclidean distance of 10 in the FAME analysis has been proposed as an indication of species-level differences (MIDI). By this standard, the liquid-evolved lineages have evolved into a new species in only 1000 generations. This interpretation is unreasonable, and so one might be tempted to suggest that the experimental populations became contaminated by some other species, but this possibility is rejected by molecular genetic analyses that show all the evolved lines, from both liquid and surface regimes, to be derived from ancestral TFD41 (Nakatsu et al., 1998). In our view, the idea that such differences in FAME composition reflect species-level differences is evidently flawed, at least as a general proposition.

\section{Concluding remarks}

Considering all of the phenotypic traits together, we find remarkable both the extent of change from the ancestral condition and the diversity among the derived lines. It is tempting to suggest that all this diversity represents different adaptive solutions achieved by each line in response to the selective environment. An alternative explanation is that a substantial fraction of the phenotypic diversity was the result of non-adaptive genotypic 
changes that spread by hitchhiking (linkage) with beneficial mutations. To the extent that the selective environment was stressful and thereby mutagenic to the bacteria, this explanation becomes more likely, as the opportunity for neutral and even deleterious mutations to hitchhike becomes greater the higher the genomic mutation rate. It has been shown that some Ralstonia strains undergo substantial stress-induced changes in their genomes, including deletions and rearrangements (Taghavi et al., 1997), and similar kinds of events were seen in this evolution experiment with strain TFD41 (Nakatsu et al., 1998). Indeed, recent evidence indicates that Ralstonia strain TFD41 undergoes deletions similar to those found in the evolved lines when subjected to certain stresses, and these deletions occur at high frequency (T. L. Marsh, unpublished data), so that they could spread by hitchhiking. We do not know whether batch culture (including lag and stationary phases, as well as growth) on 2,4-dichlorophenoxyacetate represented a comparable stress, but it is certainly possible that stress-induced genotypic changes contributed to the phenotypic diversity observed (Finkel \& Kolter, 1999). We are currently assessing the extent to which stressinduced changes in the TFD41 genome may have caused changes in cell morphology and other traits during the evolution experiment as well as determining the molecular bases of the increase in relative fitness exhibited by the derived lineages at 1000 generations.

\section{ACKNOWLEDGEMENTS}

The authors thank the Center for Electron Optics at Michigan State University for assistance with the electron microscopy. Special thanks to Carol Flegler, Uwe Danielowitz and Sally Burns. We are grateful to the Kellogg Biological Station for assisting with the FAME analysis and for the excellent technical assistance of Tracy Simpson and Elizabeth Seymoure. This work was supported by the Center for Microbial Ecology through National Science Foundation grant DEB-9120006.

\section{REFERENCES}

DeFlaun, M. F., Tanzer, A. S., McAteer, A. L., Marshall, B. \& Levy, S. B. (1990). Development of an adhesion assay and characterization of an adhesion-deficient mutant of Pseudomonas fluorescens. Appl Environ Microbiol 56, 112-119.

Doetsch, R. N. (1981). Determinative methods of light microscopy. In Manual of Methods for General Bacteriology, pp. 21-33. Edited by P. Gerhardt. Washington, DC: American Society for Microbiology.

Dykhuizen, D. E. (1990). Experimental studies of natural selection in bacteria. Annu Rev Ecol Syst 21, 373-398.

Dykhuizen, D. E. (1993). Chemostats used for studying natural selection and adaptive evolution. Methods Enzymol 224, 613-631.

Finkel, S. E. \& Kolter, R. (1999). Evolution of microbial diversity during prolonged starvation. Proc Natl Acad Sci USA 96, 4023-4027.

Fletcher, M. (editor) (1996). Bacterial Adhesion: Molecular and Ecological Diversity. New York: Wiley-Liss.

Guckert, J. B., Carr, G. J., Johnson, T. D., Hamm, B. G., Davidson, D. H. \& Kumagai, Y. (1996). Community analysis by Biolog: curve integration for statistical analysis of activated sludge microbial habitats. J Microbiol Methods 27, 183-197.

Hahn, M. W., Moore, E. R. B. \& Höfle, M. G. (1999). Bacterial filament formation, a defense mechanism against flagellate grazing: is growth rate controlled in bacteria of different phyla? Appl Environ Microbiol 65, 25-35.

Helling, R. B., Vargas, C. N. \& Adams, J. (1987). Evolution of Escherichia coli during growth in a constant environment. Genetics 116, 349-358.

Hrebenda, J., Heleszko, H., Brzostek, K. \& Bielecki, J. (1985). Mutation affecting resistance of Escherichia coli K12 to nalidixic acid. J Gen Microbiol 131, 2285-2292.

Jürgens, K., Pernthaler, J., Schalla, S. \& Amann, R. (1999). Morphological and compositional changes in a planktonic bacterial community in response to enhanced protozoan grazing. Appl Environ Microbiol 65, 1241-1250.

Korona, R. (1996). Adaptation to structurally different environments. Proc Roy Soc Lond B 263, 1665-1669.

Korona, R., Nakatsu, C. H., Forney, L. J. \& Lenski, R. E. (1994). Evidence for multiple adaptive peaks from populations of bacteria evolving in a structured habitat. Proc Natl Acad Sci USA 91, 9037-9041.

Krieg, N. R. \& Holt, J. G. (editors) (1984). Bergey's Manual of Systematic Bacteriology, vol. 1. Baltimore: Williams \& Wilkins.

Lenski, R. E. (1995). Evolution in experimental populations of bacteria. In Population Genetics of Bacteria, pp. 193-215. Edited by S. Baumberg and others. Cambridge: Cambridge University Press.

Lenski, R. E. \& Mongold, J. A. (2000). Cell size, shape and fitness in evolving populations of bacteria. In Scaling in Biology, pp. 221-235. Edited by J. H. Brown \& G. B. West. Oxford: Oxford University Press.

Lenski, R. E. \& Travisano, M. (1994). Dynamics of adaptation and diversification: a 10,000-generation experiment with bacterial populations. Proc Natl Acad Sci U S A 91, 6808-6814.

Lenski, R. E., Mongold, J. A., Sniegowski, P. D., Travisano, M., Vasi, F., Gerrish, P. J. \& Schmidt, T. M. (1998). Evolution of competitive fitness in experimental populations of E. coli: what makes one genotype a better competitor than another? Antonie Leeuwenhoek 73, 35-47.

Mongold, J. A., Bennett, A. F. \& Lenski, R. E. (1996). Experimental investigations of evolutionary adaptation to temperature. In Animals and Temperature: Phenotypic and Evolutionary Adaptation, pp. 239-264. Edited by I. A. Johnston \& A. F. Bennett. Cambridge: Cambridge University Press.

Nakatsu, C. H., Korona, R., Lenski, R. E., De Bruijn, F. J., Marsh, T. L. \& Forney, L. J. (1998). Parallel and divergent genotypic evolution in experimental populations of Ralstonia sp. J Bacteriol 180, 4325-4331.

Nikaido, H. (1996). Outer membrane. In Escherichia coli and Salmonella, pp. 29-47. Edited by F. C. Neidhardt. Washington, DC: American Society for Microbiology.

Ophir, T. \& Glutnick, D. (1994). A role for exopolysaccharides in the protection of microorganisms from desiccation. Appl Environ Microbiol 60, 740-745.

Papadopoulos, D., Schneider, D., Meier-Eiss, J., Arber, W., Lenski, R. E. \& Blot, M. (1999). Genomic evolution during a 10,000generation experiment with bacteria. Proc Natl Acad Sci U S A 96, 3807-3812.

Parke, D., D’Argenio, D. A. \& Ornston, L. N. (2000). Bacteria are not what they eat: that is why they are so diverse. J Bacteriol 182, 257-263. 
Rainey, P. B. \& Travisano, M. (1998). Adaptive radiation in a heterogeneous environment. Nature 394, 69-72.

Roberts, I. S. (1996). The biochemistry and genetics of capsular polysaccharide production in bacteria. Annu Rev Microbiol 50, 285-315.

Sasser, M. (1997). MIDI technical note 101 (revised 1997). Newark, DE: MIDI.

Simoni, S. F., Harms, H., Bosma, T. M. P. \& Zehnder, A. J. B. (1998). Population heterogeneity affects transport of bacteria through sand columns at low flow rates. Environ Sci Technol 32, 2100-2105.

Sniegowski, P. D., Gerrish, P. J. \& Lenski, R. E. (1997). Evolution of high mutation rates in experimental populations of Escherichia coli. Nature 387, 703-705.

Taghavi, S., Mergeay, M. \& van der Lelie, D. (1997). Genetic and physical maps of the Alcaligenes eutrophus $\mathrm{CH} 34$ megaplasmid pMOL28 and its derivative pMOL50 obtained after temperatureinduced mutagenesis and mortality. Plasmid 37, 22-34.

Tonso, N. L., Matheson, V. G. \& Holben, W. E. (1995). Polyphasic characterization of a suite of bacterial isolates capable of degrading 2,4-D. Microb Ecol 30, 3-24.

Travisano, M. \& Lenski, R. E. (1996). Long-term experimental evolution in Escherichia coli. IV. Targets of selection and the specificity of adaptation. Genetics 143, 15-26.

Ward, J. H. (1963). Hierarchical grouping to optimize an objective function. J Am Stat Assoc 58, 236-244.
Whitfield, C. \& Valvano, M. A. (1993). Biosynthesis and expression of cell-surface polysaccharides in Gram negative bacteria. Adv Microb Physiol 35, 136-246.

Williams, V. \& Fletcher, M. (1996). Pseudomonas fluorescens adhesion and transport through porous media are affected by lipopolysaccharide composition. Appl Environ Microbiol 62, 100-104.

Wrangstadh, M., Szewzyk, U., Östling, J. \& Kjelleberg, S. (1990). Starvation-specific formation of a peripheral exopolysaccharide by a marine Pseudomonas sp. strain S9. Appl Environ Microbiol 56, 2065-2072.

Wright, S. (1932). The roles of mutation, inbreeding, crossbreeding and selection in evolution. In Proceedings of the Sixth International Congress of Genetics, pp. 356-366. Edited by D. F. Jones. Menasha, WI: Brooklyn Botanic Garden.

Wright, S. (1988). Surfaces of selective value revisited. Am Nat $131,115-123$.

Wyndham, R. C. (1986). Evolved aniline catabolism in Acinetobacter calcoaceticus during continuous culture of river water. Appl Environ Microbiol 51, 781-789.

Yamagishi, J.-I., Yoshida, H., Yamayoshi, M. \& Nakamura, S. (1986). Nalidixic acid-resistant mutations of the gyrB gene of Escherichia coli. Mol Gen Genet 204, 367-373.

Received 20 June 2000; revised 20 November 2000; accepted 1 December 2000. 